Int. J. Speleol. 4 (1972), pp. 1-7.

\title{
Le Cinquantenaire du Premier Institut de Spéléologie du Monde
}

\author{
Traian ORGHIDAN
}

Directeur actuel de 1'Institutul de Spéologie Emil Racovitza*

L'Institutul de Spéologie 'Emil Racovitza' est arrivé en 1970 dans sa cinquantième année d'activité.

Fondé par Racovitza à Cluj en 1920, l'Institut de Spéologie fut - surtout pendant sa première décennie d'activité - le centre mondial de la Spéléologie, grâce à la petite équipe de chercheurs, dont les noms étaient particulièrement connus dans le domaine de la recherche souterraine: Emil Racovitza, René Jeannel, Pierre-Alfred Chappuis.

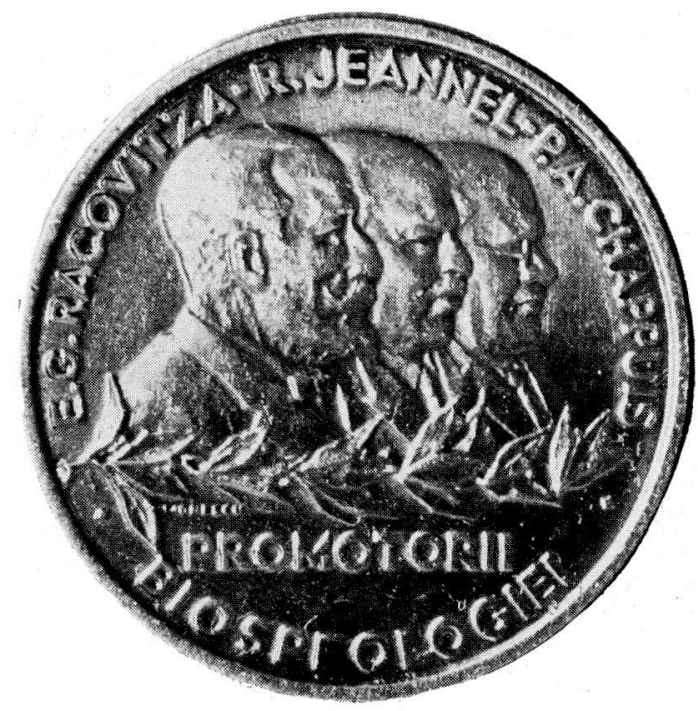

La crise économique mondiale qui s'installa après 1930 frappa aussi l'Institut de Spéologie de Cluj. Jeannel revint en France et Racovitza, surchargé de toutes sortes d'activités administratives (Recteur de l'Université, Président de l'Académie roumaine etc.) fut obligé d'assister avant sa mort à la diminution progressive de la spéléologie en Roumanie, qui, après le départ de Chappuis, ne fut plus représentée que par un seul chercheur officiel, Assistant à l'Institut de Spéologie, l'Ingénieur M. Serban.

Mais par contre, peu de temps avant sa disparition, Racovitza assiste à la renaissance des recherches spéologiques en Roumanie et surtout à l'étude de la

\footnotetext{
* 8 Rue Dr. Capsa, BUCURESTI-35-Roumanie.
} 
faune des eaux interstitielles des vallées, débutée par Chappuis en 1940 et continuée avec beaucoup de succès par le Professeur C. Motas et ses collaborateurs ( $\mathbf{J}$. Tanasachi et moi-même), à partir de 1945 .

Peu de temps avant la mort de Racovitza, D. Coman avec deux de mes anciens collègues de la Faculté des Sciences Naturelles de Bucarest, M. Serban et R. Givulesco, entreprirent, en compagnie du juriste Max Pop, l'exploration de la grotte de Scarisoara, et éclaircirent le problème de la glacière naturelle. Le rapport qu'ils ont présenté à Racovitza a dû enchanter le grand savant car cette glacière constituait pour lui une ancienne préoccupation.

Après le départ de Chappuis en 1949 la direction de l'Institut fut assurée, pour presque trois années, par le Professeur Vasile Radu, Membre Correspondant de l'Académie.

Le Comité Géologique, présidé par l'Académicien Georges Macovei, nous a demandé en 1950 de constituer deux équipes pour la prospection des dépôts de chiroptérite et phosphorite des grottes de Roumanie.

M.V. Puscariu, actuellement Secrétaire de la Commission pour la Protection de la Nature, ancien chercheur et élève de Racovitza à l'Institut de Spéologie de Cluj, et moi avons alors constitué deux équipes: l'une à Bucarest (constituée par Mme le Dr. Margareta Dumitresco, titulaire de la Chaire de Zoologie de Vertébrés à l'Université de Bucarest, notre collaboratrice Mme le Dr. Jeanne Tanasachi, M.V. Puscariu et moi) et l'autre à Cluj (composée par D. Coman, M. Serban, P. Banarascu, I. Viehmann).

Les résultats obtenus pendant les cinq années de travail de ces deux équipes, furent tels que le Président de la République, le Docteur Petru Groza, ayant en grande estime la personnalité de Racovitza et son oeuvre, procéda à la réorganisation de la recherche du domaine souterrain.

C'est ainsi qu'en 1956, par un décret spécial, le centre de la recherche spéléologique fut déplacé à Bucarest, celui de Cluj devenant une section de ce centre et le Professeur C. Motas fut nommé par le gouvernement, Directeur de 'l'Institut de Spéologie de la République populaire roumaine', qui en 1958 prend son nom actuel: 'Institutul de Spéologie Emil G. Racovitza'.

C'est ainsi que toute une nouvelle école de biospéologie se constitua en Roumanie - surtout à Bucarest - dans un court délai, suivant la tradition si fertile que Racovitza et Jeannel avaient laissée à la nouvelle génération.

Le cinquantenaire de l'Institut de Spéologie de Roumanie devait être fêté en 1970, mais les inondations, transformées par les forces déchaînées de la Nature en une véritable calamité nationale, nous ont empêché d'organiser ces manifestations, que nous dûmes remettre pour 1971.

Sous le haut patronage du Président du Conseil des Ministres, Monsieur l'Académicien Ion Gheorghe Maurer, qui avait chargé le Professeur Mircea Malita, Ministre de l'Enseignement, de donner lecture de son message adressé aux spéléologues, la séance solennelle d'ouverture eut lieu le 2 octobre 1971 à 9 h, 30 , dans la salle de l'Académie de la République Socialiste de Roumanie. Le Président de l'Académie, le Professeur Miron Nicolesco prit la parole après le message du Président du Conseil, pour évoquer lui aussi la personnalité de Racovitza et pour 
féliciter ses héritiers en leur souhaitant la continuation des succès dans leur activité scientifique.

Deux rapports ont suivi, dont l'un du Prof. Radu Codreanu, Membre correspondant de l'Académie et Valeriu Puscariu et l'autre des Docteurs Traian Orghidan et Dan Coman, pour faire le bilan de l'activité de l'Institut de Spéologie dans sa première phase (1920-1947) et dans la deuxième, (1947-1970).

C'est le Professeur Claude Delamare-Deboutteville qui, en prenant ensuite la parole, a évoqué tout d'abord les personnalités des anciens dirigeants de la Biospeologica pour porter ensuite son attention aux problèmes de l'avenir de la spéléologie.

Pour terminer le Professeur C. Motas présenta son exposé sur la féconde collaboration entre Racovitza et Jeannel.

La séance solennelle, à laquelle assistait aussi son Excellence M. Pierre Pelen, 1 'Ambassadeur de France, accompagné par Monsieur Salle, Premier Attaché Culturel, ainsi que l'Attaché Culturel de Cuba, fut suivie par une visite à l'exposition spéléologique, installée à l'occasion du cinquantenaire à la Bibliothèque de l'Académie.

Les spécialistes de l'étranger qui ont répondu aux invitations et qui ont pu assister à la séance solennelle, furent M. le Professeur Paul Brien de l'Académie royale de Belgique, M. le Professeur Bernard Gèze de l'Institut Agronomique et Président de l'Union Internationale de Spéléologie, M. le Professeur Claude DelamareDeboutteville du Muséum, Directeur du Laboratoire souterrain C.N.R.S. de Moulis et Directeur du Laboratoire d'Ecologie de Brunoy, M. Christian Juberthie, Sousdirecteur de Recherches C.N.R.S. à Moulis, M. Jean-Paul Henry de la Faculté des Sciences de Dijon représentant Mr. le Professeur Roger Husson, Rédacteur en chef de l'International Journal of Speleology, M. le Professeur Vladimir Panos, de l'Université Olomouc (Prague), Vice-president de l'Union Internationale de Spéologie, M. Geraldo Acevedo, Directeur des relations étrangères de l'Académie des Sciences de Cuba.

La même journée à $17 \mathrm{~h}, 30$ les participants aux manifestations du cinquantenaire ont rendu hommage au Professeur C. Motas, Homme de science émérite, Directeur de l'Institut de Spéologie Emil Racovitza pendant la période 1956-1964, à l'occasion de son 80ème anniversaire. La séance fut présidée par le Professeur Stefan Milou, Vice-Président de l'Académie, qui ouvrit la séance en évoquant la personnalité du Professeur Motas. Le Professeur Sergiu Carausu de l'Université de Iasi, le Docteur Mihai Bacescu, Directeur du Musée d'Histoire Naturelle Gr. Antipa de Bucarest, Mme Adriana Murgoci, Professeur à la Faculté des Sciences Naturelles de Bucarest, le Professeur Nicolae Botnariuc, Doyen de la même Faculté et Traian Orghidan, tous des anciens élèves du Professeur Motas, ont évoqué sa très marquante personnalité, ainsi que son oeuvre riche et variée. Le soir, un banquet à la 'Maison des scientifiques', pendant lequel on a entendu des dizaines de toasts, nous a permis de fêter cet anniversaire dans un cadre plus intime.

Le 4 octobre, les invités, les chercheurs de Bucarest, ainsi que des participants de différentes universités de Roumanie sont arrivés à Cluj où, par une séance solennelle à la Bibliothèque de l'Université, fut ouvert le Colloque National de Spéléologie. Le 
Président du Comité exécutif du Conseil Populaire départemental de Cluj, M. Aurel Duca, ainsi que le Recteur de 1'Université de Cluj, M.M. Pascu et le Président de la Section de Biologie de l'Académie, le Professeur Emil Pop, ainsi que le Dr. Dan Coman, Chef du secteur Cluj de l'Institut, l'Académicien Paul Brien de Belgique et Traian Orghidan, ont prononcé des discours dédiés aux manifestations du cinquantenaire, ainsi qu'à l'activité du secteur de Cluj de l'Institut de Spéologie.

Après la séance solennelle et après avoir visité l'exposition photographique, organisée par les chercheurs de Cluj de l'Institut Emil Racovitza, les participants se sont séparés et les travaux du Colloque commencèrent en deux sections simultanées: Biospéologie à la salle de la Bibliothèque de l'Université et Spéléologie physique, à l'amphithéatre de la Faculté de Géographie.

Il ne s'agît pas de nous occuper dans cet article de chaque travail qu'on a présenté à ce colloque. Il convient mieux d'extraire tout d'abord quelques traits généraux des séances et nous allons chercher ensuite à parcourir brièvement, le contenu des nombreux travaux qui ont été présentés.

A mon avis la caractéristique principale des séances, et du Colloque en général, fut la participation active - et je dirai même très active, par rapport aux séances de la Section Biospéologie des IIIe, IVe, Ve Congrès International auxquels j'ai assisté.

Cette caractéristique fut due à l'atmosphère intime qui s'est établie dès le commencement entre les invités étrangers et les participants locaux et surtout à l'attention que des Maîtres de la Zoologie et de Biospéologie actuelle, tels que les Professeurs Paul Brien et Claude Delamare-Deboutteville ont accordée aux travaux des jeunes chercheurs roumains. En présidant les séances, tous les deux, ainsi que nos collègues Monsieur et Madame Juberthie, ont su extraire la partie la plus originale, la plus intéressante de chaque travail en faisant toujours le raccord entre les contributions originales des chercheurs et les problèmes de la Biologie générale. Ainsi l'horizon de chaque travail apparaissait plus large et les problèmes plus complexes, ce qui a beaucoup incité les chercheurs à prendre la parole lors des discussions, qui se sont déroulées dans une ambiance amicale et constructive à la fois.

La même caractéristique s'est retrouvée aux séances de la Section de Spéléologie physique. Mais ici elle fut due surtout à l'esprit de combativité toujours plus accusé chez les karstologistes que chez les biospéléologues. En dehors de cela il faut reconnaître que le groupe que formait l'assistance et les auteurs qui exposaient des travaux était moins homogène que dans l'autre section. La participation des chercheurs appartenant à plusieurs unités de travail (comme: Institut géologique, Institut de géologie-géographie, Faculté de géographie et Faculté de géologie de l'Université de Bucarest, Institut de spéologie avec les chercheurs de Cluj et de Bucarest), a provoqué des discussions qui parfois furent même très animées.

Nous allons passer en revue tout d'abord quelques travaux présentés à la Section de Spéléologie physique. Nous commencerons par le travail de notre très distingué collègue, le Professeur Vladimir Panos de l'Université d'Olomouc (Tchecoslovaquie) Vice-Président de U.I.S. sur le karst récifal côtier et les récifs barrière de Cuba. M. Vladimir Panos a eu la chance de pouvoir étudier pendant des visites répétées à Cuba les formations karstiques, parfois en compagnie de notre ami Otokar Stelkl et 
aussi avec le Président de l'Académie des Sciences de Cuba, Antonio NunezJimenez.

Dans le travail présenté, M.V. Panos a réussi à décrire et à expliquer très clairement le karst côtier à Cuba, dont les caractéristiques sont dues non seulement aux particularités de la roche (récifs de Coraux et d'Algues pliocènes et pléistocènes) mais surtout aux conditions climatiques particulières (zone tropicale à régime temporairement humide) auxquelles s'ajoutent les conditions du régime littoral.

Le Professeur Gheorghe Pop et notre jeune collègue Ch. Racovitza (ce dernier a participé à la première expédition biospéologique roumano-cubaine à Cuba en 1969) ont présenté quelques observations sur les 'mogotes', formations karstiques de type conique.

Les particularités des formations karstiques de Cuba qui, pour ceux qui sont habitués au karst d'Europe, peuvent leur paraître comme extraordinaires, ont suscité des discussions après les deux communications et ont fait rêver à Cuba toute l'assistance de la section.

Le Professeur T. Naum a présenté ensuite un travail par lequel il a essayé d'extraire les caractéristiques du karst des Carpates orientales où les formations karstiques sont assez variées vu les roches, elles aussi très variées (calcaire, grès, marnes, tufs etc.).

Le même auteur en collaboration avec le Professeur I. Preda (tous les deux sont professeurs à l'Université de Bucarest) a présenté une communication sur une très intéressante capture karstique découverte dans la partie N-W des Monts Apuseni.

Notre collègue V. Senco de l'Institut de Géographie de Bucarest a présenté ses recherches sur la circulation des eaux karstiques dans la zone minière de la ville d'Anina (Banat).

Le chercheur Th. Rusu, appartenant au secteur de Cluj de l'Institut de Spéologie, a présenté les conclusions de son étude sur les vallées karstiquesdu massif de Padurea Craiului. L'accent a été mis sur l'évolution des vallées karstiques ce qui a provoqué des intéressantes discussions.

Notre distingué collègue Valeriu Puscariu nous a presenté une note sur la grotte 'Pestera de la Despicatura', récemment découverte à Baile Herculane dans la zone des célèbres sources thermales - grotte tapissée par des cristaux de gypse et dont la température atmosphérique atteint au fond $51^{\circ} \mathrm{C}$ !

Quelques précisions nouvelles quant à la structure minéralogique de cristaux de calcite de la grotte 'Pestera Muierilor' (Olténie) furent apportées par un travail présenté par notre jeune collègue Gabriel Diaconu de l'Institut 'E. Racovitza', en collaboration avec Horst Peter de l'Institut de Prospections Géologiques de Bucarest. L'étude optique de coupes minces ont mis en évidence l'existence de la pseudomorphose de la calcite sur l'aragonite.

Ch. Racovitza a présenté ensuite un essai sur les aspects multiples du phénomène de la glaciation dans la glacière naturelle de Scarisoara, en partant d'une analyse statistique des caractères des hivers pour une période de 750 ans. L'auteur a réussi d'établir des rapports quantitatifs entre l'évolution climatique et la dynamique des dépôts souterrains de glace auxquels il a ajouté les données obtenues par l'étude de la structure du massif de glace. 
Le Professeur I. Vintilescu a donné un aperçu sur les devoirs actuels de la recherche en spéologie physique et sur les perspectives dans ce domaine.

D'autres rapports et études ont été présentés par les chercheurs de Cluj sur la grotte de Meziad (Bihor), sur les dépôts alluvionnaires de grottes des montagnes Padurea Craiului, sur la classification de la morphologie du karst souterrain et sur l'expédition roumano-tchécoslovaque aux Mts Apuseni en 1971 etc.

Le caractère constructif des travaux de cette section s'est cristallisé à la fin par des conclusions, consignées sous forme d'un procès verbal, signé par tous les participants aux séances, dans lequel ont été fixées quelques directions d'activité dans le but d'une meilleure corrélation des études de tous les spécialistes qui travaillent en Roumanie dans le domaine de la Spéléologie physique.

La section de Biospéologie a commencé ses travaux au même moment que la section de Spéléologie physique dans la matinée du 4 octobre. Les communications ont commencé par l'exposé du travail du Professeur Claude Delamare, en collaboration avec notre chercheur E. Serban sur le genre Austrobathynella. Contrairement à l'opinion de W. Noodt - qui en 1964 a mis en synonymie le genre Austrobathynella avec Bathynella - l'etude plus approfondie des caractères chez l'espèce $A$. patagonica Delamare, démontre que l'entité du genre Austrobathynella est réelle malgré la ressemblance - quant à l'habitus - avec le genre Bathynella .

Un rapport sur l'ensemble des recherches sur la faune souterraine de Madagascar envoyé par notre collègue $M$. Remillet a été exposé par le chercheur V. Decou. En s'occupant de la faune des Coléoptères endogés. M. Remillet nous a fait connaître qu'on a trouvé à Madagascar des espèces dont l'affinité avec des espèces africaines est manifeste tandis que d'autres ont subi une spéciation plus poussée qu'en Afrique.

Le Professeur Codreanu, en collaboration avec Doina Balcesco, nous a présenté ensuite un travail sur la distribution des Planaires souterrains en Dobrogea; il s'agit des espèces du genre Dendrocoelium dont la répartition semble être fonction de la transgression sarmatienne.

Un exposé dû à L. Botsaneanu et V. Decou s'est occupé du fameux 'essai' de E.G. Racovitza. Les auteurs ont entrepris une analyse du remarquable travail, en concluant que la valeur de la conception de Racovitza donne à cette oeuvre aujourd'hui encore, après 65 ans de recherches, la qualification d'un véritable 'statut fondamental de la Biospéologie'.

Le Docteur Dan Coman a présenté ensuite une étude sur les Mermithides hyporhéophiles d'Europe, d'Afrique et de Cuba, tandis que le chercheur F. Botea nous présentait un travail à caractère écologique sur les Oligochètes hyporhéophiles de la vallée de Crisul Repede (Mts Apuseni).

M-me A. Negrea a exposé un travail sur le polymorphisme du système d'ornementation chez le Gastéropode Cepaea vindobonensis de Roumanie.

M-me Lisiane Juberthie-Jupeau nous a présenté une analyse des conditions d'élevage des espèces de Troglocaris au Laboratoire souterrain de Moulis.

M-me Maria Georgesco nous a décrit le développement postembryonnaire chez l'Araignée Diplocephalus cristatus; Dan Danielopol a présenté ses recherches sur la morphologie et la répartition des Ostracodes Enthocythérides de la tribu des Sphaeromicolini. 
Les Myriapodes ont été l'objet d'études présentées par T. Ceuca, Z. Matic, Prunesco.

Le Professeur S.I. Ljovusckin de Moscou nous a envoyé un travail sur les Tréchines de l'U.R.S.S.

Le chercheur V. Decou nous a parlé de ses premières études sur les Coléoptères Leptotyphlites de Cuba.

Un très intéressant mémoire a été exposé par M. Jean-Paul Henry de Dijon, délégué par le professeur Roger Husson pour représenter son groupe de chercheurs au cinquantenaire. Nous avons pu avoir ainsi une connaissance détaillée des dernières recherches biospéologiques du Laboratoire de Biologie Animale et Générale de la Faculté des Sciences de Dijon.

D'autres mémoires ont été présentés comme celui de I. Tabacaru, sur les Isopodes Trichoniscides de Roumanie, celui de D. Dancau sur les Amphipodes souterrains de Majorque, de Gh. Racovitza sur la dynamique des populations des Coléoptères cavernicoles, de M-mes M. Gruia et A. Zamfirescu sur les variations saisonnières de la faune de Collemboles.

La section de paléontologie de l'Institut de Spéologie 'E. Racovitza' a été représentée par E. Terzea, C. Radulesco et P. Samson, qui ont présenté des travaux sur les Mammifères quaternaires de Roumanie, tandis que I. Viehmann nous a parlé de quelques traces de l'Ours des cavernes dans les grottes de Roumanie.

MM G. Racovitza et C. Plesa, nous ont présenté une étude sur le milieu spécial que les ours représentent pour la vie de certaines espèces aquatiques souterraines.

Enfin, le chercheur L. Gruia de Sinaia a présenté une étude sur quelques Algues des grottes de Roumanie.

D'autres travaux ont été envoyés de l'étranger pour les faire imprimer dans le livre du cinquantenaire.

Tout d'abord il s'agit du raport du Professeur René Ginet sur l'activité de recherche de l'équipe 'Biologie souterraine' de l'Université de Lyon.

C'est le moment pour moi de mentionner que la riche participation de la recherche biospéologique française au Colloque du Cinquantenaire de l'Institut Racovitza, nous a fourni le plaisir d'entendre d'excellentes communications de ceux qui sont les premiers dans la recherche biospéologique actuelle.

En même temps il faut reconnaître aussi que l'on peut s'apercevoir de plus en plus de l'intensité croissante avec laquelle se manifeste ces derniers temps, la collaboration franco-roumaine dans ce domaine et notre satisfaction est grande.

L'ambiance intime, extrêmement amicale, qui s'est manifestée au Colloque a continué durant les excursions en Moldavie et au Delta du Danube.

La médaille du jubilé représentant les profils des promoteurs de la Biospéologie, E.G. Racovitza, René Jeannel et Pierre Alfred Chappuis, symbolise la réussite de la légendaire 'Biospeologica'. En même temps elle rend hommage à la collaboration franco-roumaine dans un domaine particulier de recherches qui devrait fournir un heureux exemple pour la coopération internationale. 